\title{
A Weber-like law for perceptual learning
}

\author{
Andrew T. Astle', Roger W. Li², Ben S. Webb' , Dennis M. Levi² \& Paul V. McGraw'
}

SUBJECT AREAS:

PSYCHOLOGY

VISUAL SYSTEM

NEUROSCIENCE

CORTEX

Received

16 October 2012

Accepted

4 January 2013

Published

29 January 2013

Correspondence and requests for materials should be addressed to

A.T.A. (andrew.astle@ nottingham.ac.uk)
'Visual Neuroscience Group, School of Psychology, The University of Nottingham, NG7 2RD, UK, ${ }^{2}$ School of Optometry \& Helen Wills Neuroscience Institute, University of California, Berkeley, CA 94720, USA.

What determines how much an organism can learn? One possibility is that the neural factors that limit sensory performance prior to learning, place an upper limit on the amount of learning that can take place. We tested this idea by comparing learning on a sensory task where performance is limited by cortical mechanisms, at two retinal eccentricities. Prior to learning, visual performance at the two eccentricities was either unmatched or equated in two different ways (through spatial scaling or visual crowding). The magnitude of learning was equivalent when initial levels of performance were matched regardless of how performance was equated. The magnitude of learning was a constant proportion of initial performance. This Weber-like law for perceptual learning demonstrates that it should be possible to predict the degree of perceptual improvement and the final level of performance that can be achieved via sensory training, regardless of what cortical constraint limits performance.

$\mathrm{t}$ is well established that repeated practice can improve the sensory abilities of a mature organism ${ }^{1}$. These improvements can range from simple sensory discriminations in the laboratory (e.g. distinguishing the displacement of one line from another) to discriminations of more complex sensory patterns in the real world (e.g. assessing x-ray images for abnormalities) ${ }^{2,3}$. Much less clear however, is the precise nature of the environmental and biological constraints that dictate how much an organism can learn. Understanding, and potentially removing, these obstacles to learning could open up new avenues for learning-based therapeutic interventions - an approach proving to be extremely useful for recovering and treating different forms of sensory loss in mature organisms (e.g. $\left.{ }^{4-6}\right)$.

We can quantify the visual abilities of an organism as a psychophysically measured visual threshold - the smallest amount of change in a visual stimulus that produces a measurable sensation. Any visual stimulus consists of information that can potentially be used by an organism to perform a given task. But it's ability to use that information is modulated by extraneous fluctuations in the physical environment (e.g. low light levels, heavy rain, dense fog) and internal fluctuations within its central nervous system (e.g. sampling density of the photoreceptor array, stochastic nature of neural firing, the modulation of activity from adjacent neurons) ${ }^{7}$. It is these environmental and biological factors that ultimately place limits on our perception of the visual world. A visual threshold quantifies the performance of the visual system when it is operating at or near these limits.

We asked whether the same factors that set fundamental limits to visual thresholds also constrain the amount an organism can learn on a visual task? Thresholds typically increase (i.e. visual performance deteriorates) when visual stimuli are presented to increasingly peripheral retinal locations, largely because of coarser sampling by the photoreceptor and ganglion cell array and their pattern of projection to visual cortex ${ }^{8}$. If initial threshold levels determine the magnitude of learning, it follows that equating thresholds at different retinal eccentricities prior to learning should lead to equivalent amounts of learning. We tested this hypothesis by comparing the amount of learning in human subjects on a Vernier alignment task with stimuli presented at two different retinal eccentricities ( 5 and 15 degrees). Vernier thresholds are cortically limited ${ }^{9,10}$ and can be rendered the same at peripheral eccentricities by spatially scaling stimuli to produce cortical representations of equivalent size ${ }^{10,11}$. They can also be impaired and elevated at peripheral eccentricities by visual crowding - a phenomenon in which nearby stimuli have a deleterious effect on visual performance ${ }^{12}$. We compared the amount of learning when alignment thresholds were different or equated at the two retinal eccentricities either by spatially scaling or visually crowding stimuli.

We find that equating thresholds before learning leads to equivalent amounts of learning, regardless of how thresholds were equated. Where thresholds were different before learning, either due to retinal eccentricity, size of the stimulus, crowding, or inter-subject variability, we find learned improvements to be proportional to the performance level prior to training. We show that this relationship holds over a very wide range of performance levels for a large number of subjects. Additionally, we show that trained improvements transfer to a retinal location at which no training is undertaken, and that the degree of transfer is also linked to the initial performance at that location. 


\section{Results}

We first compare the amount of learning when visual thresholds before learning were unmatched. Eleven subjects trained on a Vernier alignment task with stimuli of identical retinal size, presented separately at 5 and 15 degrees eccentricity ('unscaled' - top row Figure 1). To ascertain whether any improvements in performance transferred to an untrained retinal location, alignment thresholds were also measured at a location midway between the trained locations (10 degrees eccentricity) on the first and final training sessions To facilitate interpretation of the data and comparison between different groups, we expressed subjects' performance as a Weber fraction (Vernier threshold/line length) throughout, unless otherwise stated. Lower Weber fractions indicate better performance.

Figure 2 shows data for subjects who trained with stimuli of identical retinal size (unscaled). In this and subsequent figures, red, green and blue symbols indicate data collected with stimuli presented at 5, 10 and 15 degrees eccentricity. It is well known that Vernier thresholds increase with eccentricity ${ }^{13}$, most likely due to an increase in neural convergence and decrease in cortical magnification ${ }^{11,14}$ (physical extent of visual cortex devoted to a given angular extent of visual space $\left.^{15}\right)$. Consistent with this notion, we found performance in the first session was worse for more eccentric locations (see Figure 2a). Mean $( \pm S D)$ Weber fractions were $0.10( \pm 0.03), 0.18( \pm 0.07)$ \& $0.27( \pm 0.07)$ at 5,10 and 15 degrees eccentricity, respectively. An ANOVA (see Data analysis) revealed a significant effect of eccentricity on initial performance level $[\mathrm{F}(2,10)=40.58, \mathrm{p}=<.0001]$, with post-hoc comparisons indicating statistical differences in subjects' performance at all three eccentricities.

The improvements in Vernier alignment performance over the course of training (expressed as the difference in Weber fractions between the first and last session) (see Figure 2c), were 0.03 ( \pm 0.02 ) and $0.15( \pm 0.09)$ at the trained eccentricities of 5 and 15 degrees, respectively. At the untrained (10 degree) eccentricity, performance improved by $0.05( \pm 0.08)$. There was a statistically significant effect of eccentricity on the improvements $[\mathrm{F}(2,10)=10.37, \mathrm{p}=<.001]$. Post-hoc analysis indicated a significant difference in improvement at an eccentricity of 15 degrees compared to at 5 or 10 degrees. The performance change that occurred with eccentricity mirrored the differences in subjects' threshold before learning. In other words,

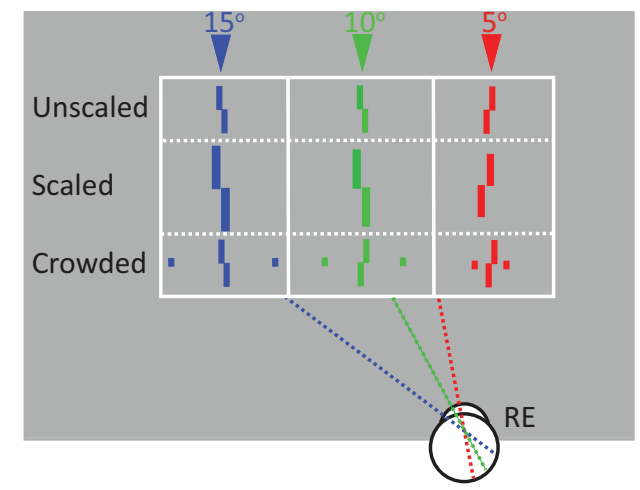

Figure 1 Schematic illustration of stimuli. Stimuli were always white and presented on a black background, but are shown here and represented in subsequent figures in red (5 deg), green (10 deg) and blue (15 deg). Unscaled stimuli (top row) were the same retinal size at each eccentricity; scaled stimuli (middle row) were larger at more eccentric locations; crowded stimuli (bottom row) were the same size at each eccentricity. As crowding increases with retinal eccentricity, different spatial distances were required between target and flanker to equate performance. Subjects were trained with stimuli presented at 5 and 15 degrees. Vernier alignment threshold at 10 degrees was only measured on the first and last training sessions. we found that the magnitude of improvements in Vernier alignment performance were proportional to initial levels of performance.

Matching performance by scaling stimuli equates the magnitude of learning. To investigate this relationship further, in separate groups of participants, we altered the stimuli at each eccentricity to equate performance before training and measured improvements following otherwise identical periods of training. We first equated performance by spatially scaling stimuli. We adopted a procedure used by Whitaker et al., $(1992)^{11}$ to spatially scale stimuli. In two subjects (who did not undergo training) Vernier thresholds were measured as a function of line length at the fovea and at 3 eccentric locations: 5, 10 and 15 degrees in the nasal peripheral field of the right eye. Results were expressed as Weber fractions and plotted relative to stimulus line length. This created a series of curves, each horizontally displaced along the abscissa (Figure 3a). For each eccentricity, a scaling factor was determined that minimised the mean squared error between foveal and eccentric data ${ }^{11}$. There was excellent agreement between data collected in the present study and that collected previously by Whitaker and colleagues, (see Figure 3b). We fitted a linear regression to the scaling factors obtained at each eccentricity to determine the size of stimuli required to equate peripheral to foveal alignment performance. The size of stimuli required was 1.09, 1.91 and 2.73 degrees at 5, 10 and 15 degree eccentricities, respectively. The resultant scaling factors reflect the fact that Vernier alignment thresholds increase with eccentricity and that correspondingly larger eccentric stimuli are required to equate peripheral to foveal performance levels.

Eleven new subjects trained with stimuli that were magnified using the scaling procedure described above. Therefore, stimuli were of different retinal size (larger for greater eccentricities), but were equated in terms of cortical size at each eccentricity (see middle row of Figure 1). Figure 4a shows alignment performance in the first session at different eccentricities: scaled stimuli successfully matched performance at each eccentricity. Mean Weber fractions in the first session were $0.033( \pm 0.01), 0.034( \pm 0.01) \& 0.036( \pm 0.01)$ at 5,10 and 15 degrees, respectively. There were no statistical differences in Weber fraction at each eccentricity at the start of training $[F(2,10)=$ $0.59, \mathrm{p}=0.56]$. Initial performance at 5 degrees was better for subjects trained with scaled stimuli (relative to unscaled stimuli) as scaling equates performance relative to the fovea and stimulus magnification is introduced for all eccentricities beyond this.

Figure $4 \mathrm{~b}$ shows mean learning curves at 5 and 15 degrees and performance on the first and last sessions at 10 degrees. Improvements in performance (expressed as above) were 0.013 $( \pm 0.008)$, and $0.020( \pm 0.01)$ at the trained eccentricities of 5 and 15 degrees. At the 10 degree location, where no training was received, performance improved by $0.019( \pm 0.02)$. There were no statistical differences in the amount of improvement at each eccentricity $[\mathrm{F}(2,10)=2.082, \mathrm{p}=0.15]$. Put simply, learning was equivalent at each eccentricity when alignment performance was equated before training.

Matching performance by crowding stimuli equates the magnitude of learning. To investigate whether this finding was specific to the method used to change performance (spatial scaling), we used a different approach and matched alignment performance in the first session by introducing variable amounts of crowding. Flanking target stimuli by presenting additional lines in nearby locations degrades performance - a phenomenon known as crowding. The spatial extent over which crowding occurs grows with eccentricity ${ }^{10}$. It is therefore possible to match performance at different eccentricities by placing flankers at different spatial distances from the stimuli (see Figure 1 bottom row).

To determine the exact flanker to target distances required to equate performance at each eccentricity, Vernier alignment thresholds were measured in four subjects at 5, 10 and 15 degrees as a 
a.

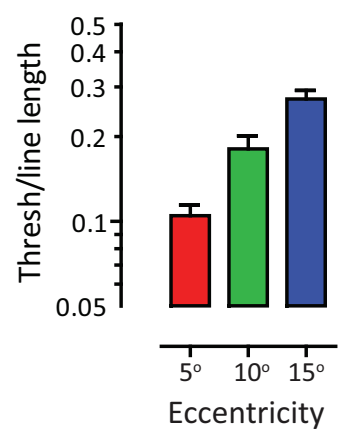

b.

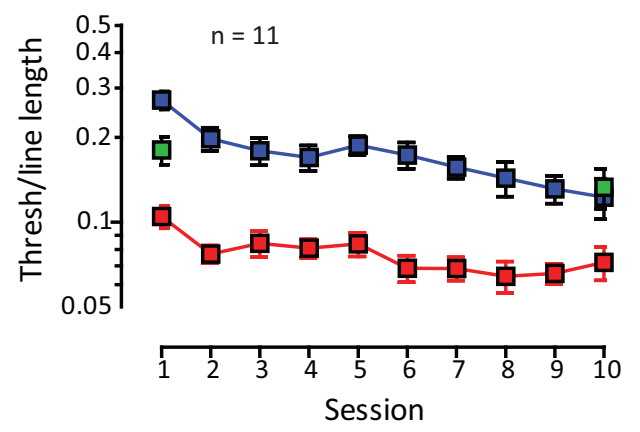

C.

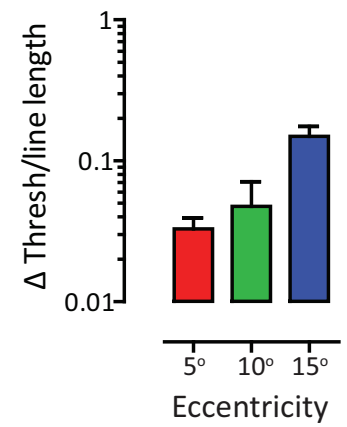

Figure $2 \mid$ Learning with unscaled stimuli. Performance on session 1 (a) at 5, 10 and 15 degrees. (b) Mean learning curves for 11 subjects at 5 and 15 degrees. Data for 10 degrees, where no training took place, is shown for session 1 and 10. (c) Improvement in performance at each eccentricity. Improvement is quantified as the difference between performance on the first and final sessions. Data for 5, 10 and 15 degrees are shown in red, green and blue, respectively. Initial performance drops with increases in retinal eccentricity. However, improvement in performance generated by training on the task across 10 sessions increases with retinal eccentricity. Error bars represent 1 standard error of the mean (SEM).

function of flanker distance (the distance of the flanker to the centre of the target lines). Target stimuli of the same size as the unscaled stimuli ( $0.275 \mathrm{deg}$ at each eccentricity) were crowded with flankers $1 / 4$ the length of each line element. Results are plotted in figure 5 where the curve fits are best fitting solutions of Equation 1 (see Data anlysis). Flanker distances were chosen from each curve that corresponded to the same performance level (see yellow lines). This gave flanker distances of approximately 70, 240 and 550 minutes of arc for eccentricities of 5, 10 and 15 degrees eccentricities.

Eleven new subjects trained with stimuli that were crowded by flankers; lower eccentricities required smaller flanker distances to equate performance. Figure $6 \mathrm{a}$ shows alignment performance in the first session at each eccentricity. The introduction of flankers with predetermined separations successfully matched initial performance levels at each eccentricity (mean Weber fractions: 0.38 ( \pm 0.09$), 0.44$ ( \pm 0.12$) \& 0.41( \pm 0.14)$ at 5,10 and 15 degrees). There was no significant effect of eccentricity on performance at the start of training $[\mathrm{F}(2,10)=3.05, \mathrm{p}=0.07]$.

Mean improvements in performance were $0.13( \pm 0.06)$, and $0.17( \pm 0.10)$ at the trained eccentricities of 5 and 15 degrees and $0.14( \pm 0.10)$ at the untrained location. There was no significant effect of eccentricity on improvement $[\mathrm{F}(2,10)=1.67, \mathrm{p}=0.21]$. Therefore, equating performance before training using crowding led to equivalent amounts of improvement at each eccentricity.

a.



Magnitude of learning is inversely proportional to initial performance levels. Where thresholds in the first session did not statistically differ, improvements in performance were not statistically different. Similarly, in every case where starting performance was statistically different, we found a statistical difference in the magnitude of performance change (see Figures 2,4 \& 6). Results from subjects who trained with scaled and crowded stimuli provide compelling evidence that threshold gains are tightly coupled to performance levels prior to training, regardless of the way in which threshold is constrained. When stimuli were spatially scaled, thresholds for more eccentric locations were reduced. In contrast, for crowded stimuli, performance was equated at a much higher threshold level. Thus, the relationship between learned improvements and threshold before training holds over a very large performance range.

Figure 7 shows improvements plotted against starting threshold. When quantified in terms of the difference in Weber fraction between first and final session (Figure 7a), there is a proportional relationship: the higher the starting threshold the greater the improvement. The correlation of improvement and start performance in Fig $7 \mathrm{a}$ is significant $\left(\mathrm{r}^{2}=0.60, \mathrm{P}<.0001\right)$. Learning data is often presented as a ratio between starting and finishing performance and when expressed in this way (and plotted against performance in the first session; see Figure $7 \mathrm{~b}$ ), subjects improved by a factor of b.

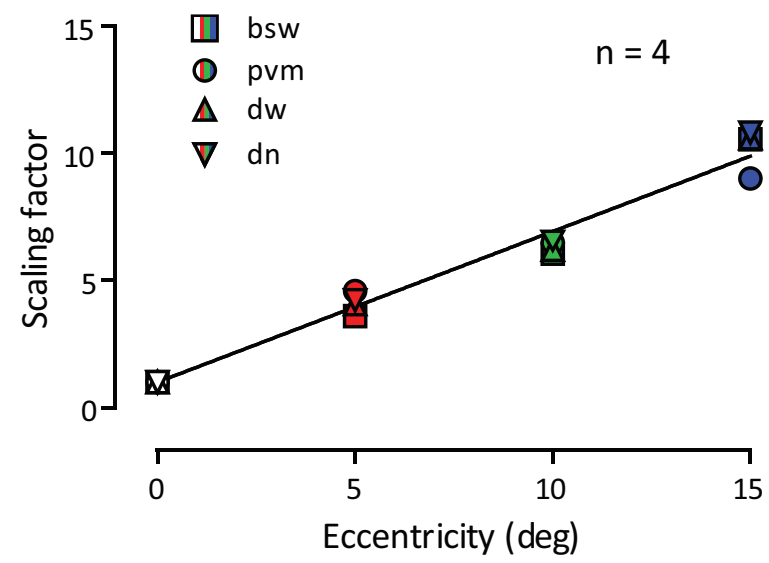

Figure 3 Spatially scaling stimuli equates peripheral to foveal Vernier alignment thresholds. Weber fractions for the fovea and 3 eccentric locations are plotted (data shown in lower contrast) as a function of line length for a representative subject. (a) A scaling factor that minimised the mean squared error between the eccentric and foveal data (see high contrast data points) was calculated. (b) A linear regression line fitted through the data estimates the scaling factor required to increase the size of the eccentric stimuli such that performance is matched to that at the fovea. dw and dn are data reproduced from Whitaker et al., (1992) ${ }^{11}$. 
a.

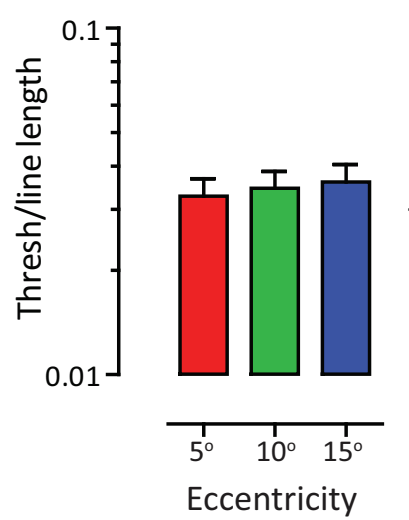

b.

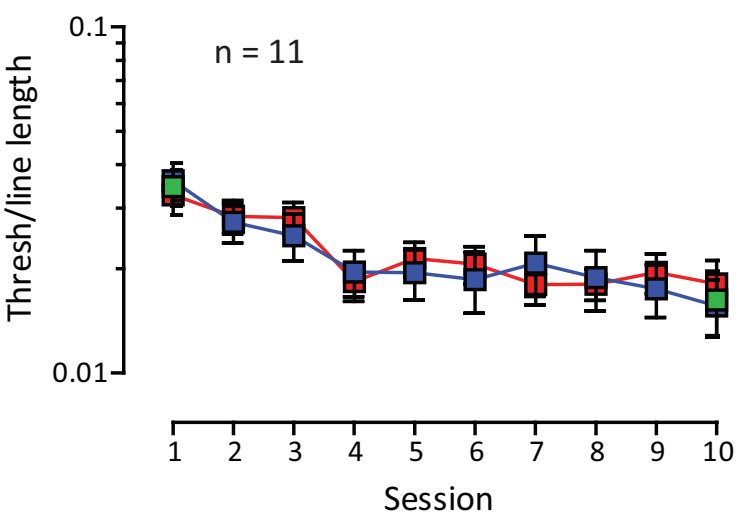

c.

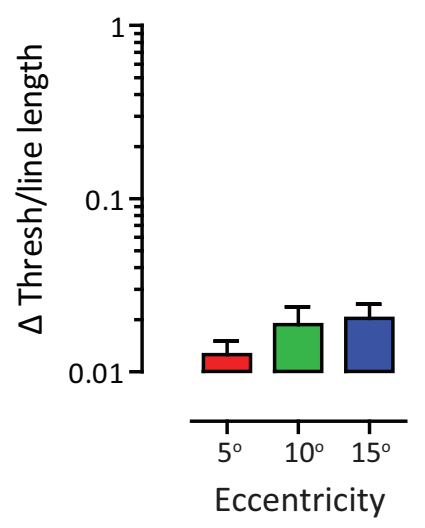

Figure $4 \mid$ Learning with spatially scaled stimuli. (a) Performance on session 1 at 5, 10 and 15 degrees. (b) Learning curves showing mean performance on each session at 5 and 15 degrees. Data for 10 degrees, where no training took place, is shown for session 1 and 10 . (c) Improvement in performance at each eccentricity. Improvement is quantified as the difference between performance on the first and final sessions. Data for 5,10 and 15 degrees is shown in red, green and blue, respectively. Performance at each eccentricity is the same on session 1 and there is no significant difference in improvement at each eccentricity. Error bars represent 1 standard error of the mean (SEM).

$2.18( \pm 1.53)$, on average. The slope of the curve fitted to the data in Figure $7 \mathrm{~b}$ is -0.116 and does not differ significantly from zero $[\mathrm{F}(1,97)=3.74, \mathrm{p}=0.06]$. Data for the 10 degrees location are included in addition to the trained locations and show that a substantial amount of learning transfer takes place. Performance improved by a factor of $2.13( \pm 1.78)$ at 10 degrees and was not significantly different to that measured at the trained locations $(2.20( \pm 1.41)$. The improvement at $10 \mathrm{deg}$ is unlikely to be the result of simply taking two repeat measurements at that location, since at the trained locations, improvements between any two consecutive sessions (e.g. 1 and 2) were much smaller (see figures 2, 4 \& 5). Additionally, at 5 and 15 degrees, threshold improvements are evident over the course of training, rather than being driven by specific changes occurring early or late in the training schedule.

To assess the reliability of applying a performance improvement factor of 2.18 to predict final performance from start performance, actual performance was plotted against predicted performance on session 10 (Figure 7c). Predicted performance was calculated by

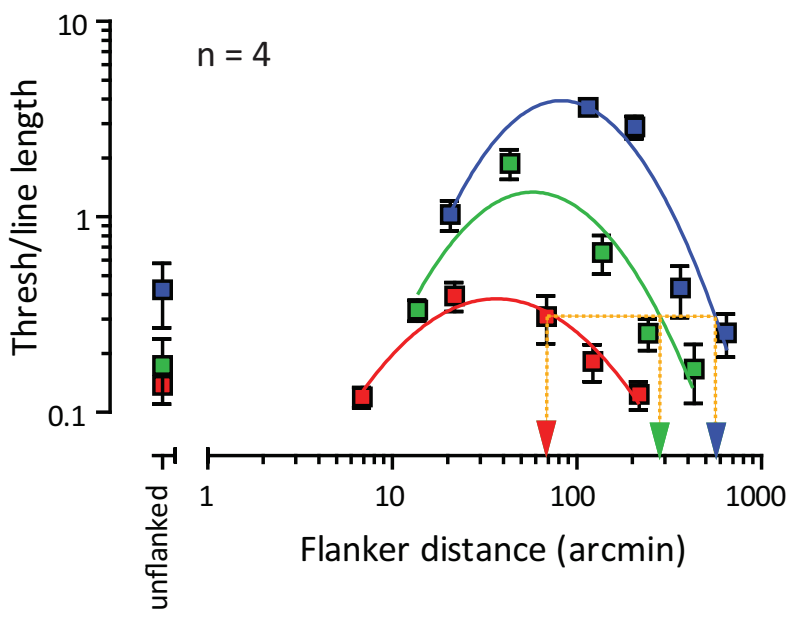

Figure $5 \mid$ The effect of flanker distance on Vernier alignment at each eccentricity. Mean vernier threshold/line length at 5 (red), 10 (green) and 15 (blue) degrees plotted for 4 subjects as a function flanker distance. Smooth curves are best fitting solutions of Equation 1. Flanker distances were estimated from each curve that corresponded to the same threshold (yellow lines). dividing the performance on session 1 by 2.18 . The slope of the resultant curve fitted in Figure $7 \mathrm{c}$ is $0.98\left(\mathrm{r}^{2}=0.79\right)$ and does not differ significantly from unity $[\mathrm{F}(1,97)=0.07, \mathrm{p}=0.79]$.

We found no statistical differences between the age of subjects in each group $[F(2,30)=1.218, p=0.31]$ and no statistically significant relationship between the age of subjects and the magnitude of improvements in performance $([\mathrm{F}(1,97)=3.64, \mathrm{p}=0.06]$ extra sum-of-squares F test).

\section{Discussion}

Our results provide strong evidence that learned improvements in visual thresholds are strongly coupled to performance levels prior to training. Equating performance by spatially scaling stimuli, or by introducing different levels of crowding, leads to equivalent levels of learning - something that would be predicted if improvements are dependent on pre-training performance. As a result of the systematic variation in performance created by performing the task at different retinal eccentricities, with different sized stimuli, with different levels of crowding, not to mention the small natural individual variation between observers, we were able to faithfully characterize the relationship between improvements and initial performance levels. Poor initial thresholds levels lead to more improvement and vice versa. Weber's law states that the just-noticeable difference between two stimuli (in this case the separation between two Vernier elements) is proportional to the intensity of the stimuli. Our results show that perceptual learning also obeys a similar Weber-like law, whereby improvements generated during a fixed period of training are proportional to starting threshold.

Several studies have demonstrated improvements in Vernier acuity following practice ${ }^{16-19}$. Fundamental changes to the neural processing of spatial information required to successfully perform the task $\mathrm{k}^{20}$, as opposed to learning associated with better knowledge of the task procedure, are thought to mediate the improvements. In support, practice-based improvements in Vernier thresholds are strongly coupled to the orientation of the test lines ${ }^{17,21}$. Additionally, changes in oculo-motor control (e.g. increased accuracy of accommodation or fixation stability) that could regulate retinal image quality are unlikely to underlie these effects since Vernier thresholds are relatively insensitive to image blur ${ }^{22}$ and motion $^{23,24}$. As in several previous studies ${ }^{16,17,18,25}$, we found some inter-subject variability in the amount of improvement following training. This has been attributed to factors such as previous experience, attention, general levels of arousal, age, intelligence, motivation 
a.

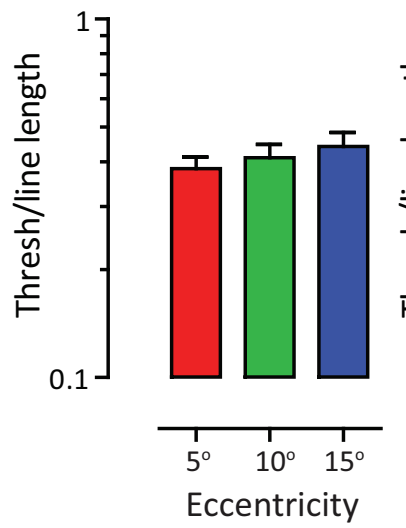

b.

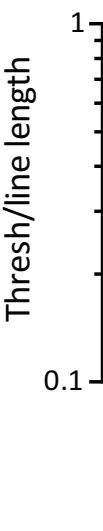

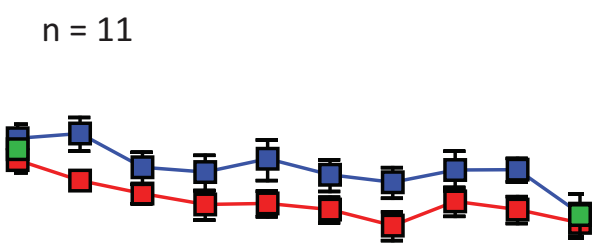

1



c.

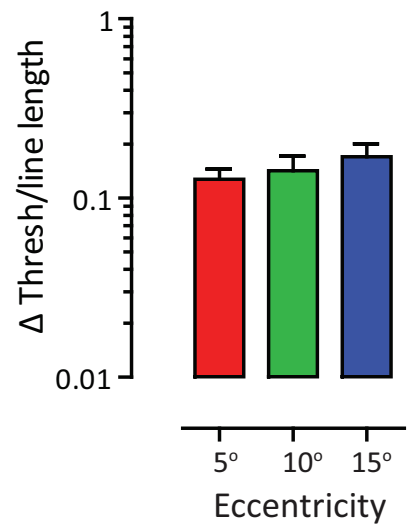

Figure 6 Learning with crowded stimuli. (a) Performance on session 1 at 5, 10 and 15 degrees. (b) Learning curves showing mean performance on each session at 5 and 15 degrees, with data for 10 degrees shown for the first and final session. (c) Improvement in performance at each eccentricity. Performance at each eccentricity is the same on session 1 and there is no significant difference in the magnitude of improvement across eccentricities. Error bars as before.

etc $^{18}$. We show that, despite this variability, it is possible to predict future performance from start performance for the vast majority of individuals.

Previous studies have shown that perceptual learning-based improvements are influenced by initial levels of performance in subjects with normal vision ${ }^{18,26-29}$. Fahle \& Henke-Fahle ${ }^{18}$ reported greater improvements when starting performance was poorer. However, subjects were all trained at the fovea and the small variations in Vernier threshold evident prior to training reflected normal levels of inter-observer variability. It has been suggested that this individual variation in threshold is related to variations in the size of primary visual cortex $(\mathrm{V} 1)^{9}$. We show that the relationship between starting threshold and level of improvement is robust and operates over a range three orders of magnitude larger than that investigated in the Fahle \& Henke-Fahle study. Furthermore, as we trained each subject concurrently at two peripheral retinal locations our results confirm that the relationship applies to within-subject as well as between-subject comparisons of learning.

Spatially scaling stimuli to account for changes in cortical magnification results in equivalent levels of learning and transfer of learning at different eccentricities. Previous work that has examined learning at different eccentricities reported results that were highly specific to the learning task ${ }^{30}$. For example, subjects could learn to discriminate grey-scale images (compound Gabor patches) just as well in two locations (fovea and at $3 \mathrm{deg}$.) when stimuli were spatially scaled, but category learning retained a scaleinvariant advantage at the fovea ${ }^{30}$. This suggests that category learning may be functionally distinct from basic visual discriminations and is a task that requires mechanisms beyond those that encode elementary visual features such as orientation or relative position. The fact that category learning appears to be a foveal specialization, may ultimately limit the ability of this form of learning to generalize to other retinal locations. We show that when the spatial judgment remains constant, equating learning is not specific to accounting for eccentricity-dependent changes in cortical representation per se, but can be achieved regardless of the method used to set threshold. Certainly, for tasks that are limited by the sampling resolution of neurons in early visual cortex (V1), such as Vernier alignment, it does not matter if the associated neural uncertainty results from the eccentricity at which the task is performed, the size of the stimulus, the amount of visual clutter around the stimulus or individual differences in absolute sensitivity to relative spatial position. This suggests that whatever mechanism is reading out this position information, it is agnostic to the source of sensory uncertainty but not its magnitude.
We found complete transfer of improvements to 10 degrees eccentricity, where no training was undertaken. This was rather surprising given that it has previously been shown that learning on Vernier acuity tasks is highly location specific ${ }^{31}$. This could be explained by the fact that Vernier thresholds were measured for each subject at 10 degrees in the first session. Testing at one location before training and then training at a different location leads to complete transfer of learning to the tested location ${ }^{32}$, highlighting the possibility that higher non-retinotopic areas are involved in perceptual learning ${ }^{32,33}$. One study has demonstrated that, for a detection task, improvements spread to non-trained locations across a putative window of spatial attention and are not limited to target locations ${ }^{34}$. Therefore, we cannot rule out the possibility that the 10 degree location in the present study was situated within such a window induced by training at locations 5 degrees either side, resulting in transfer to this non-trained location. Although it has previously been assumed that specificity for orientation, spatial frequency or location pointed towards the locus of learning residing in the neural networks that encode such information, equally possible is the proposal ${ }^{35}$ that what is specifically learnt is how best to efficiently decode the relevant visual information to improve the accuracy of discrimination judgments. This could occur more centrally and need not require functional changes in the tuning preferences of individual neurons. The transfer of learning to an untrained location, and the fact that internal neural noise seems to have a common influence on learning would seem to be broadly consistent with this view.

Our results suggest that it is possible to predict the amount of improvement and level of performance ultimately obtainable following training in normal subjects. In the present study we show that improvements on a trained task are a constant proportion of the initial threshold level. That is to say, when improvements are expressed as a ratio between performance on the first and final day of training, they are constant - as has previously been found ${ }^{36,37}$. On average, individuals improved by a factor of approximately two. If the rate of improvement for each initial performance level is the same throughout the training period, the relationship between improvement and start performance will remain fixed. The relationship will breakdown, however, if a floor or saturation level in improvement is reached earlier for individuals starting at a particular threshold level compared to other starting levels. Theoretically, this could be caused by a variety of factors depending on the task. For example, retinal photoreceptor spacing may limit improvements in resolution threshold past some optimum level. Additionally, some studies have continued training for long periods of time and found subsequent 



Predicted thresh/line length on final session

Figure 7 Improvement in performance as a function of starting performance. Improvement at 5 (red), 10 (green) and 15 degrees (blue), expressed as the difference in performance between the first and final session. (a) The curve shows best fitting solutions Equation 2 with a slope 1.007 and intercept -0.42 . Improvements are a constant proportion (approximately 0.38 ) of starting performance. (b) Improvements plotted as a ratio between the final and first session. (c) Measured final session performance plotted as a function of final session performance as predicted by applying a universal improvement factor of 2.18 .

cascades of performance improvements after an initial plateau has been reached ${ }^{38}$. Therefore, the time period over which improvements are considered may influence the relationship between start performance and improvement.

The present study was not designed to investigate the effects of external noise on learning. Despite this, the results are in general agreement with studies that have found consistent levels of improvement across different levels of external noise ${ }^{39,40}$. There are however exceptions. For example, no improvement occurs at low external noise levels following extensive practice on a foveal orientation identification $\operatorname{task}^{41}$, and for texture-defined stimuli, improvements occur predominantly at low external noise conditions ${ }^{42}$. Therefore, depending on the nature of the task or stimulus, the magnitude of learning can differ when different amounts of external noise is added to the stimulus ${ }^{42}$.

Previous work ${ }^{38,43,44}$ has demonstrated that improvements following training are linked to deficit levels prior to training in individuals with amblyopia - a developmental anomaly of spatial vision. However, in contrast to our findings in subjects with normal vision, some studies investigating perceptual learning in amblyopia show that improvements expressed as a ratio are greater when initial performance levels are worse ${ }^{38,45,46}$. This suggests that either the mechanisms driving the performance gain or the factors limiting threshold in amblyopia are different. In support of this, subjects with amblyopia have been shown to improve on tasks where those with normal vision do not (e.g. $\left.{ }^{43,45,47,48}\right)$. Although the amblyopic visual system is limited 
Table 1 Subject information. Information for all subjects trained. Subjects are divided into those that trained with stimuli of the same size (unscaled), those that trained with stimuli which were scaled to match initial performance (scaled) and those that trained with crowded stimuli to match initial performance (crowded). $M=$ male, $\mathrm{F}=$ female, $\mathrm{OD}=$ right eye, $\mathrm{DS}=$ dioptre sphere, $\mathrm{Pl}=$ plano, $\mathrm{VA}=$ visual acuity, $\log M A R=$ logarithm of the minimum angle of resolution

Subject Age Gender OD Distance Rx VA (LogMAR)

$\begin{array}{lcccc}\text { Unscaled } & & & & \\ \text { JJJ } & 22 & \mathrm{~F} & -3.25 /-0.50 \times 180 & -0.10 \\ \text { JC } & 22 & \mathrm{~F} & -3.25 /-1.25 \times 165 & -0.06 \\ \text { MD } & 24 & \mathrm{~F} & -3.50 \mathrm{DS} & -0.10 \\ \text { AY } & 24 & \mathrm{M} & -2.25 /-1.50 \times 165 & -0.10 \\ \text { RT } & 22 & \mathrm{M} & \mathrm{Pl} & -0.12 \\ \text { GW } & 23 & \mathrm{M} & -3.00 /-2.00 \times 178 & -0.14 \\ \text { WL } & 22 & \mathrm{~F} & -0.25 \mathrm{DS} & -0.08 \\ \text { QT } & 29 & \mathrm{~F} & -1.25 /-0.25 \times 105 & -0.08 \\ \text { JJ } & 23 & \mathrm{~F} & \mathrm{Pl} & -0.10 \\ \text { RL } & 41 & \mathrm{M} & -4.50 \mathrm{DS} & -0.10 \\ \text { LL } & 29 & \mathrm{M} & -1.50 \mathrm{DS} & -0.10\end{array}$

Spatially scaled

\begin{tabular}{lcccc}
\hline ATA & 30 & $M$ & $-0.50 \mathrm{DS}$ & -0.12 \\
ZH & 31 & $\mathrm{~F}$ & $\mathrm{Pl}$ & -0.10 \\
HS & 21 & $\mathrm{M}$ & $-0.25 \mathrm{DS}$ & -0.12 \\
JL & 21 & $\mathrm{M}$ & $\mathrm{Pl} /-0.25 \times 30$ & -0.10 \\
GH & 22 & $\mathrm{~F}$ & $+0.25 \mathrm{DS}$ & -0.14 \\
ID & 20 & $\mathrm{~F}$ & $-0.75 /-0.50 \times 100$ & -0.12 \\
KR & 20 & $\mathrm{M}$ & $-0.25 /-0.50 \times 100$ & -0.12 \\
AS & 20 & $\mathrm{~F}$ & $+0.25 \mathrm{DS}$ & -0.12 \\
ADP & 21 & $\mathrm{~F}$ & $\mathrm{Pl}$ & -0.02 \\
SO & 20 & $\mathrm{~F}$ & $+3.00 /-0.50 \times 165$ & 0.00 \\
PB & 20 & $\mathrm{M}$ & $+0.25 \mathrm{DS}$ & 0.00
\end{tabular}

Crowded

\begin{tabular}{lcccc}
\hline KB & 25 & $\mathrm{~F}$ & $-0.25 /-0.50 \times 55$ & -0.08 \\
ZH & 21 & $\mathrm{M}$ & $\mathrm{Pl}$ & -0.16 \\
$\mathrm{OO}$ & 26 & $\mathrm{~F}$ & $\mathrm{Pl} /-0.25 \times 115$ & -0.14 \\
$\mathrm{AP}$ & 24 & $\mathrm{~F}$ & $-0.50 \mathrm{DS}$ & -0.14 \\
BS & 30 & $\mathrm{~F}$ & $\mathrm{Pl}$ & -0.10 \\
$\mathrm{CLY}$ & 36 & $\mathrm{~F}$ & $-3.00 \mathrm{DS}$ & -0.08 \\
JPW & 21 & $\mathrm{~F}$ & $-0.25-0.25 \times 95$ & -0.14 \\
AT & 22 & $\mathrm{~F}$ & $-0.50 /-0.50 \times 165$ & -0.06 \\
EC & 27 & $\mathrm{~F}$ & $-3.75 /-0.50 \times 175$ & -0.12 \\
MH & 20 & $\mathrm{~F}$ & $\mathrm{Pl} /-0.50 \times 110$ & -0.02 \\
RC & 20 & $\mathrm{M}$ & $\mathrm{Pl} /-0.50 \times 15$ & -0.12 \\
\hline
\end{tabular}

by many of the same factors that limit normal vision (e.g. external noise), additional sources of internal noise have been identified that dramatically increase levels of spatial uncertainty or markedly distort the neural representation of visual stimuli ${ }^{49-53}$. It is possible that the mechanisms that underpin visual learning must first overcome these additional constraints that elevate thresholds beyond normal levels. Indeed, learning-based reductions in contrast detection thresholds in normal subjects can be explained exclusively by external noise reduction, while improvements in amblyopic subjects require additional internal noise reduction ${ }^{54}$. Consequently, the adult amblyopic visual system may possess greater potential for improvement, which supports the therapeutic use of perceptual learning procedures in this clinical group ${ }^{46,54,55}$.

The finding that improvements in normal subjects are tied to their initial threshold in a lawful way, analogous to Weber's law, suggests that the same factors that impose limits on a visual threshold also constrain the amount an organism can learn on a visual task.

\section{Methods}

Subjects. Thirty-nine subjects with normal or corrected-to-normal vision participated in the study (mean $25 \pm 5$ years). The experimental procedures adhered to the tenets of the Declaration of Helsinki and were approved by a local ethics committee at the School of Psychology, The University of Nottingham. The refractive error of each subject was determined and visual acuity measured using a Bailey-Lovie chart $^{56}$ prior to training. Table 1 contains information for each subject who underwent training. None of the subjects had previously undergone any training on the Vernier alignment task. The mean age $( \pm S D)$ of subjects in each group was $26( \pm 6), 22( \pm 4) \& 25$ ( \pm 5$)$ years for the unscaled, scaled and crowding groups, respectively.

Apparatus. Stimuli were generated on a PC using custom software written in Python $^{57}$. Stimuli were presented on a LaCie Electron 22 Blue IV color CRT monitor (LaCie, OR, USA) with a resolution of $1920 \times 1440$ and a refresh rate of $85 \mathrm{~Hz}$. Subjects had their heads held in position using a forehead and chinrest and testing was carried out in a dark room. Fixation was maintained on a small illuminated fixation dot positioned to the right side of the monitor. Stimuli were presented at the centre of the screen and eccentricity controlled by altering the viewing distance. The viewing distances were $1,1.5 \& 3$ meters for eccentricities of 15,10 and 5 degrees, respectively.

Psychophysical measurements: Stimuli and task Stimuli consisted of vertically oriented abutting Vernier lines. The luminance of the Vernier lines was $83 \mathrm{cdm}^{-2}$ and the luminance of the background $0.29 \mathrm{cdm}^{-2}$. Stimuli were presented at 5, 10 or 15 degrees in the nasal periphery of the right eye, whilst the left eye was occluded with a patch. On each trial, either the top or bottom Vernier line was randomly assigned to placement at the required eccentricity. The top Vernier line was positioned to the left or to the right relative to the bottom line in random sequence from trial to trial.

Stimuli were presented for $200 \mathrm{msec}$ on each trial.

In a two-alternative forced choice task, subjects were required to indicate whether the top Vernier line was displaced to the left or to the right of the bottom Vernier line with a key press. Auditory feedback was given at the end of each trial. If the top line was displaced to the left relative to the bottom line, a low-pitched tone (frequency $=$ $300 \mathrm{~Hz}$ ) was played. If the top line was displaced to the right relative to the bottom line, a high-pitched tone (frequency $=800 \mathrm{~Hz}$ ) was played. The next stimulus was displayed immediately after the subject's response. The horizontal separation of the two Vernier lines was varied using a 3-down, 1-up staircase procedure. Staircases terminated after 60 trials. Thresholds were estimated as the mean of the last 4 reversals.

Training paradigm. All subjects completed 10 sessions. Each session was carried out on a different day. Where possible, sessions were completed on consecutive days and there was a gap of no more than three days between any two sessions for each observer. On the first and final sessions, Vernier alignment thresholds were measured at 5, 10 and 15 degrees. At each eccentricity, threshold was measured using a single staircase of 60 trials. On sessions 2-8, subjects trained at 5 and 15 degrees. At each eccentricity, 5 randomly interleaved staircases (60 trials in each) were used to measure threshold.

Thirty-three subjects were divided into 3 groups. The first group trained with stimuli which had the same angular size (height of each line $=0.275$ degrees) at each eccentricity i.e. unscaled. The second and third groups trained with stimuli that were manipulated to equate initial performance (threshold/line length on Session 1) at each eccentricity; by spatially scaling stimuli and by crowding, respectively. Figure 1A shows a schematic of the stimuli used at 5, 10 and 15 degrees for each of the 3 groups.

Data analysis. To establish whether there were any statistical differences in subject's alignment performance at different eccentricities before, during and after learning, we used a one-way repeated measures ANOVA and Tukey HSD test for post-hoc comparisons.

Weber fractions plotted as a function of flanker-target distance at different eccentricities (Figure 5), were fitted with a log Gaussian function of the form:

$$
W=A \times \exp \left(-0.5 \times\left(\ln (d / C) / \sum\right)^{2}\right)
$$

where $W$ is threshold/line length, $A$ is amplitude, $d$ is flanker distance, $C$ is the centre of the distribution and $\sum$ is the width of the distribution. The relationship between improvement in performance and initial levels of performance in all conditions at each eccentricity (Figure $7 \mathrm{a}, \mathrm{b}$ and $\mathrm{c}$ ) was fitted with following function:

$$
\Delta W=\exp \left(c+m \times \log \left(W_{1}\right)\right)
$$

where $\Delta W$ is the degree of improvement, $c$ is the intercept, $m$ is the slope and $W_{1}$ is the performance in the first session. The relationship between actual and predicted performance (Figure 7c) was fitted with the same equation, where $y$ is actual performance and $x$ predicted performance on the final session.

1. Gibson, E. J. Perceptual learning. Annu. Rev. Psychol. 14, 29-56 (1963).

2. Fahle, M. \& Poggio, T. Perceptual learning. (MIT Press, 2002).

3. Fine, I. \& Jacobs, R. A. Comparing perceptual learning tasks: A review. J Vis. 2, 190-203 (2002).

4. Levi, D. M. \& Li, R. W. Improving the performance of the amblyopic visual system. Philosophical Transactions of the Royal Society B: Biological Sciences. 364, 399-407 (2009). 
5. Tallal, P., Merzenich, M., Miller, S. \& Jenkins, W. Language learning impairment: Integrating research and remediation. Scand. J. Psychol. 39, 197-199 (1998).

6. Chung, S. T. L. Improving reading speed for people with central vision loss through perceptual learning. Invest Ophthalmol Vis Sci. 52, 1164-1170 (2011).

7. Faisal, A. A., Selen, L. P. J. \& Wolpert, D. M. Noise in the nervous system. Nat Rev Neurosci. 9, 292-303 (2008).

8. Strasburger, H., Rentschler, I. \& Jüttner, M. Peripheral vision and pattern recognition: A review. J Vis. 11 (2011).

9. Duncan, R. O. \& Boynton, G. M. Cortical magnification within human primary visual cortex correlates with acuity thresholds. Neuron. 38, 659-671 (2003).

10. Levi, D. M., Klein, S. A. \& Aitsebaomo, A. P. Vernier acuity, crowding and cortical magnification. Vision Res. 25, 963-977 (1985).

11. Whitaker, D., Rovamo, J., Macveigh, D. \& Mäkelä, P. Spatial scaling of vernier acuity tasks. Vision Res. 32, 1481-1491 (1992).

12. Westheimer, G. \& Hauske, G. Temporal and spatial interference with vernier acuity. Vision Res. 15, 1137-1141 (1975).

13. Westheimer, G. The spatial grain of the perifoveal visual field. Vision Res. 22, 157-162 (1982).

14. Virsu, V., Näsänen, R. \& Osmoviita, K. Cortical magnification and peripheral vision. J. Opt. Soc. Am. A. 4, 1568-1578 (1987).

15. Daniel, P. M. \& Whitteridge, D. The representation of the visual field on the cerebral cortex in monkeys. The Journal of Physiology. 159, 203-221 (1961).

16. McKee, S. P. \& Westheimer, G. Improvement in vernier acuity with practice. Percept. Psychophys. 24, 258-262 (1978).

17. Fahle, M. \& Edelman, S. Long-term learning in vernier acuity: Effects of stimulus orientation, range and of feedback. Vision Res. 33, 397-412 (1993).

18. Fahle, M. \& Henke-Fahle, S. Interobserver variance in perceptual performance and learning. Invest Ophthalmol Vis Sci. 37, 869-877 (1996).

19. Herzog, M. H. \& Fahle, M. The role of feedback in learning a vernier discrimination task. Vision Res. 37, 2133-2141 (1997).

20. Gilbert, C. D., Sigman, M. \& Crist, R. E. The neural basis of perceptual learning Neuron. 31, 681-697 (2001).

21. Poggio, T., Fahle, M. \& Edelman, S. Fast perceptual learning in visual hyperacuity. Science. 256, 1018-1021 (1992).

22. Watt, R. J. \& Hess, R. F. Spatial information and uncertainty in anisometropic amblyopia. Vision Res. 27, 661-674 (1987).

23. Westheimer, G. \& McKee, S. P. Visual acuity in the presence of retinal-image motion. J. Opt. Soc. Am. 65, 847-850 (1975).

24. Morgan, M. J., Watt, R. J. \& McKee, S. P. Exposure duration affects the sensitivity of vernier acuity to target motion. Vision Res. 23, 541-546 (1983).

25. Saarinen, J. \& Levi, D. M. Perceptual learning in vernier acuity: What is learned? Vision Res. 35, 519-527 (1995).

26. Aberg, K. C., Tartaglia, E. M. \& Herzog, M. H. Perceptual learning with chevrons requires a minimal number of trials, transfers to untrained directions, but does not require sleep. Vision Res. 49, 2087-2094 (2009).

27. Fahle, M. Specificity of learning curvature, orientation, and vernier discriminations. Vision Res. 37, 1885-1895 (1997).

28. Polat, U. et al. Training the brain to overcome the effect of aging on the human eye. Sci. Rep. 2 (2012).

29. Baldassarre, A. et al. Individual variability in functional connectivity predicts performance of a perceptual task. Proc. Natl. Acad .Sci. U. S. A. 109, 3516-3521 (2012)

30. Jüttner, M. \& Rentschler, I. Scale-invariant superiority of foveal vision in perceptual categorization. Eur. J. Neurosci. 12, 353-359 (2000).

31. Fahle, M., Edelman, S. \& Poggio, T. Fast perceptual learning in hyperacuity. Vision Res. 35, 3003-3013 (1995).

32. Zhang, T., Xiao, L.-Q., Klein, S. A., Levi, D. M. \& Yu, C. Decoupling location specificity from perceptual learning of orientation discrimination. Vision Res. $\mathbf{5 0}$, 368-374 (2010).

33. Otto, T. U., Öğmen, H. \& Herzog, M. H. Perceptual learning in a nonretinotopic frame of reference. Psychological Science. 21, 1058-1063 (2010).

34. Ahissar, M. \& Hochstein, S. The spread of attention and learning in feature search: Effects of target distribution and task difficulty. Vision Res. 40, 1349-1364 (2000).

35. Mollon, J. D. \& Danilova, M. V. Three remarks on perceptual learning. Spat. Vis. 10, 51-58 (1996)

36. Johnson, C. A. \& Leibowitz, H. W. Practice effects for visual resolution in the periphery. Percept. Psychophys. 25, 439-442 (1979).

37. Fendick, M. \& Westheimer, G. Effects of practice and the separation of test targets on foveal and peripheral stereoacuity. Vision Res. 23, 145-150 (1983).

38. Li, R. W., Klein, S. A. \& Levi, D. M. Prolonged perceptual learning of positional acuity in adult amblyopia: Perceptual template retuning dynamics. J. Neurosci. 28, 14223-14229 (2008)
39. Dosher, B. A. \& Lu, Z. L. Perceptual learning reflects external noise filtering and internal noise reduction through channel reweighting. Proc. Natl. Acad. Sci. U. S. A. 95, 13988-13993 (1998).

40. Lu, Z. L., Chu, W. \& Dosher, B. A. Perceptual learning of motion direction discrimination in fovea: Separable mechanisms. Vision Res. $\mathbf{4 6}$ 2315-2327 (2006).

41. Lu, Z.-L. \& Dosher, B. A. Perceptual learning retunes the perceptual template in foveal orientation identification. J. Vis. 4 (2004).

42. Dosher, B. A. \& Lu, Z. L. Level and mechanisms of perceptual learning: Learning first-order luminance and second-order texture objects. Vision Res. 46 1996 (2006).

43. Polat, U., Ma-Naim, T., Belkin, M. \& Sagi, D. Improving vision in adult amblyopia by perceptual learning. Proc. Natl. Acad .Sci. U. S. A. 101, 6692-6697 (2004).

44. Levi, D. M., Polat, U. \& Hu, Y. S. Improvement in vernier acuity in adults with amblyopia. Practice makes better. Invest Ophthalmol Vis Sci. 38, 1493-1510 (1997).

45. Astle, A. T., Webb, B. S. \& McGraw, P. V. The pattern of learned visual improvements in adult amblyopia. Invest Ophthalmol Vis Sci. 52, 7195-7204 (2011).

46. Astle, A. T., Webb, B. S. \& McGraw, P. V. Spatial frequency discrimination learning in normal and developmentally impaired human vision. Vision Res. 50, 2445-2454 (2010).

47. Astle, A. T., Webb, B. S. \& McGraw, P. V. The pattern of learned visual improvements in adult amblyopia. IOVS (2011, in press).

48. Hussain, Z., Webb, B., Astle, A. \& McGraw, P. Perceptual learning alleviates crowding in amblyopia and the normal periphery. J Vis. 11, 425 (2011).

49. Wang, H., Levi, D. M. \& Klein, S. A. Spatial uncertainty and sampling efficiency in amblyopic position acuity. Vision Research. 38, 1239-1251 (1998).

50. Levi, D. M., Klein, S. A. \& Sharma, V. Position jitter and undersampling in pattern perception. Vision Res. 39, 445-465 (1999).

51. Lagreze, W.-D. \& Sireteanu, R. Two-dimensional spatial distortions in human strabismic amblyopia. Vision Res. 31, 1271-1288 (1991).

52. Hess, R. F. \& Howell, E. R. The threshold contrast sensitivity function in strabismic amblyopia: Evidence for a two type classification. Vision Research. 17, 1049-1055 (1977).

53. Bedell, H. D. \& Flom, M. C. Monocular spatial distortion in strabismic amblyopia. Invest Ophthalmol Vis Sci. 20, 263-268 (1981).

54. Huang, C.-B., Lu, Z.-L. \& Zhou, Y. Mechanisms underlying perceptual learning of contrast detection in adults with anisometropic amblyopia. J Vis. 9 (2009).

55. Huang, C., Zhou, Y. \& Lu, Z. L. Broad bandwidth of perceptual learning in the visual system of adults with anisometropic amblyopia. Proc. Natl. Acad. Sci. U. S. A 105, 4068-4073 (2008).

56. Bailey, I. L. \& Lovie, J. E. New design principles for visual acuity letter charts Am. J. Optom. Physiol. Opt. 53, 740-745 (1976).

57. Peirce, J. W. Psychopy--psychophysics software in python. J. Neurosci. Methods. 162, 8-13 (2007)

\section{Acknowledgements}

The authors would like to thank Zahra Hussain for helpful discussion regarding statistical analysis of data. The research leading to this paper has received funding from the European Community's Seventh Framework Programme [FP2007-2013] under grant agreement no 223326. Ben Webb was funded by a Wellcome Trust Research Career Development Fellowship.

\section{Author contributions}

A.T.A., B.S.W. and P.V.M. wrote the main manuscript text, prepared all figures, and conducted the experiments. All authors analyzed the data and reviewed the manuscript.

\section{Additional information}

Competing financial interests: The authors declare no competing financial interests.

License: This work is licensed under a Creative Commons

Attribution-NonCommercial-NoDerivs 3.0 Unported License. To view a copy of this license, visit http://creativecommons.org/licenses/by-nc-nd/3.0/

How to cite this article: Astle, A.T., Li, R.W., Webb, B.S., Levi, D.M. \& McGraw, P.V. A Weber-like law for perceptual learning. Sci. Rep. 3, 1158; DOI:10.1038/srep01158 (2013) 